\title{
Comment on: How do paper and electronic records compare for completeness? A three centre study
}

\author{
Muhammed Omar Qadir ${ }^{1} \cdot$ Anju Kadyan ${ }^{1}$
}

Received: 23 December 2018 / Accepted: 8 April 2019 / Published online: 10 May 2019

(c) The Royal College of Ophthalmologists 2019

We congratulate $\mathrm{Wu}$ et al. on their study, as monitoring of records is important for clinical and medicolegal reasons, especially during the challenging transition towards paperless working in NHS hospitals [1-3]. However, we have concerns with their study methodology and conclusions.

New glaucoma referrals were reviewed at three sites with two different EPR systems at different stages of rollout and clinical engagement. They compared these to 'pooled' paper records from only two sites with no mention of possible use of structured paper proformas or paramedical staff collating data. Conclusions are based on data sampled possibly randomly over 5 years (2010-2015), including a changeover phase in 2014, which itself could have contributed to poorer outcomes. Separating the values for the EPR systems also provides some clues on poor quality (Table 1). Both systems may have been used simultaneously at Moorfields during transition when it was left to clinician preference as it is difficult to explain how 1 in 10 new referrals did not have eye pressures recorded. Gonioscopy recording in EPR is significantly different to paper records and, as expected, had low entries but, interestingly, did not differ between both systems.

We also tracked data quality over 1 year in our eye casualty after EPR (Medisoft) introduction, and noted consistent issues with use of free text entries due to lack of familiarity, inadequate data fields and few specified forced choice defaults. Moreover, as staff changed during this period there was a gradual deterioration in record quality (Table 2). We recommend regular electronic record audits

These authors contributed equally: Muhammed Omar Qadir,

Anju Kadyan

Muhammed Omar Qadir

Moqadir1991@gmail.com

1 University Hospitals Coventry and Warwickshire, Clifford Bridge Road Coventry, West Midlands CV2 2DX, UK with continued targeted training following mandatory induction. Feedback via user groups can facilitate software changes in future EPR versions allowing better integration with workflow.

Although $\mathrm{Wu}$ et al. rightly raise awareness on this issue, it is important to not make biased and unsupported conclusions on electronic working, e.g., electronic data is more accessible, but data breaches may not be more common, but just more easily tracked. EPR has the power to truly transform healthcare, but we need to focus on the roll out to ensure better integration with workflow to fully realize their potential.

\section{Compliance with ethical standards}

Conflict of interest The authors declare that they have no conflict of interest.

Publisher's note: Springer Nature remains neutral with regard to jurisdictional claims in published maps and institutional affiliations.

\section{References}

1. Wu CHK, Luk SMH, Holder RL, Murdoch I. How do paper and electronic records compare for completeness? A three centre study. Eye. 2018;32:1232-6.

2. Somner J, Hingorani M, Sparrow J. Electronic medical records Standards for UK Ophthalmology Services. London: The Royal College of Ophthalmologists; 2018.

3. Health and social care information centre, academy of medical royal colleges. Standards for the clinical structure and content of patient records. London: HSCIC; 2013. 
Table 1 Data review: separating the percentage entry for two EPR systems and paper across relevant fields (Wu et al.) [1]
Table 2 Summary of our data in eye casualty record quality audit (UHCW)

\begin{tabular}{llllc}
\hline & $\begin{array}{l}\text { Medisoft }(\%) \\
n=170\end{array}$ & $\begin{array}{l}\text { Open eyes }(\%) \\
n=100\end{array}$ & $\begin{array}{l}\text { Paper }(\%) \\
n=170\end{array}$ & $\begin{array}{l}\chi^{2} p \text {-values Paper versus } \\
\text { Medisoft }(\text { Open eyes })\end{array}$ \\
\hline Intraocular pressure & 98.8 & 90 & 100 & $0.50(<0.001)^{\mathrm{a}}$ \\
Central corneal thickness & 80.6 & 85 & 85.9 & $0.19(0.84)$ \\
Gonioscopy & 62.35 & 64 & 88.8 & $<0.001(<0.001)$ \\
Fundus examination & 88.8 & 69 & 90 & $0.72(<0.001)$ \\
Past medical history & 81.17 & 58 & 92.4 & $0.002(<0.001)$ \\
Current medications & 80.58 & 47 & 93.5 & $<0.001(<0.001)$ \\
Glaucoma medications & 78.8 & 28 & 88.2 & $0.19(<0.001)$ \\
Drug allergies & 78.8 & 38 & 87.6 & $0.03(<0.001)$ \\
\hline
\end{tabular}

${ }^{a}$ Fisher exact test

\begin{tabular}{lcll}
\hline & $\begin{array}{l}2016 \\
n=100\end{array}$ & $\begin{array}{l}2017 \\
n=100\end{array}$ & $\begin{array}{l}\chi^{2} p \text {-values 2016 } \\
\text { versus 2017 Medisoft entries }\end{array}$ \\
\hline Presenting complaint & 99 & 85 & $<0.001$ \\
Past ocular history & 70 & 57 & 0.06 \\
Past medical history & 63 & 38 & $<0.001$ \\
Drug history & 27 & 22 & 0.41 \\
Allergies & 49 & 21 & $<0.001$ \\
Family history & 19 & 5 & 0.002 \\
Social history & 12 & 5 & 0.04 \\
Diagnosis & 95 & 93 & 0.55 \\
Prescription recorded & 100 & 99 & $1^{\mathrm{a}}$ \\
Outcome recorded & 98 & 96 & 0.41 \\
\hline
\end{tabular}

\title{
Response to: Comment on 'How do paper and electronic records compare for completeness? A three centre study'
}

\author{
Clara Hoi Ka Wu $\mathbb{1}^{1} \cdot$ Sheila M. H. Luk ${ }^{2} \cdot$ Richard L. Holder $^{3} \cdot$ Zena Rodrigues $^{4} \cdot$ Faisal Ahmed ${ }^{4} \cdot \operatorname{lan}$ Murdoch $\mathbb{1}^{2}$
}

Received: 9 April 2019 / Accepted: 9 April 2019 / Published online: 10 May 2019

(c) The Royal College of Ophthalmologists 2019

Clara Hoi Ka Wu

clarahoika.wu@gmail.com

Whittington Health NHS Trust, London, UK

2 Moorfields Eye Hospital NHS Foundation Trust, London, UK

3 Guy's and St Thomas' NHS Foundation Trust, London, UK

4 Imperial College Healthcare NHS Trust, London, UK
To the Editor,

We thank Qadir and Kadyan for their comments concerning our study [1]. The interesting point is that the data they share shows exactly the same findings as we report. With specific reference to our methodology:

1. We sampled the data over three sites with two different EPR systems as we believe this reduced the bias of analysis based on a specific EPR. 